\title{
A stochastic time-dependent green capacitated vehicle routing and scheduling problem with time window, resiliency and reliability: a case study
}

\author{
Masoud Rabbani", Soroush Aghamohammadi Bosjin, Reza Yazdanparast and Niloufar \\ Akbarian Saravi
}

School of Industrial Engineering, College of Engineering, University of Tehran, Tehran, Iran

\begin{tabular}{l}
\hline C H R O N I C L E \\
\hline Article history: \\
Received October 2, 2017 \\
Received in revised format: \\
January 8, 2017 \\
Accepted February 26, 2018 \\
Available online \\
February 26, 2018 \\
\hline Keywords: \\
Green vehicle routing \\
Stochastic time-dependent \\
capacitated vehicle routing \\
problem \\
Scheduling \\
Time window \\
Resiliency \\
Reliability \\
\hline
\end{tabular}

\section{A B S T R A C T}

This paper presents a new multi-objective model for a vehicle routing problem under a stochastic uncertainty. It considers traffic point as an inflection point to deal with the arrival time of vehicles. It aims to minimize the total transportation cost, traffic pollution, customer dissatisfaction and maximizes the reliability of vehicles. Moreover, resiliency factors are included in the model to increase the flexibility of the system and decrease the possible losses that may impose on the system. Due to the NP-hardness of the presented model, a metaheuristic algorithm, namely Simulated Annealing (SA) is developed. Furthermore, a number of sensitivity analyses are provided to validate the effectiveness of the proposed model. Lastly, the foregoing meta-heuristic is compared with GAMS, in which the computational results demonstrate an acceptable performance of the proposed SA.

(C) 2018 by the authors; licensee Growing Science, Canada.

\section{Introduction}

The main focus in classical versions of vehicle routing problem is about finding a good solution which is able to minimize the covered distance by vehicles. The importance of this problem is more illuminating when our purpose is to deal with the perishable products, in such position a comprehensive transshipment policy can improve the benefits of the company, customer satisfaction and also decrease the possible accident which can impose different negative side effects to the system. Considering different aspects of opportunities and threats that can occur in transshipment atmosphere led us to propose a trustable model which is more compatible with practical problems. One of the most critical problems in transshipment problem is about the situation of roads an way which can be considered as a variable with high instability in developed countries due to lack of standard road conditions. One of the most important problems is about traffic thus considering a time-dependent problem can lead to a

* Corresponding author

E-mail address: mrabani@ut.ac.ir (M. Rabbani)

C 2018 by the authors; licensee Growing Science, Canada doi: $10.5267 /$ j.dsl.2018.2.002 
realistic model. One of the most important international concern is about air pollution and disadvantages of greenhouse gases thus considering the minimization of radiated gas emissions as a green goal is another aspect of this paper which have a direct relationship with the traffic issue. Combination of the proposed parameters is useful for companies and environment.

The probability of vehicle breakdowns is another inevitable problem that can cause different problems such as economic losses, Customer dissatisfaction and Loss of credibility, however; accidents are inevitable but can be decreased. Considering a predetermined flexible instruction can also decrease the disadvantages of the accident and that's the reason for considering resiliency and reliability in this paper. Resiliency is a multidimensional comprehensive instruction increasing the tolerance of a system against possible events, the main purpose of resiliency is to be prepared against accident and prevent them and also change an upset system into a normal system (Azadeh et al., 2017). Resiliency is comprised of different factors, the most justifiable factors regarding our model are Flexibility and Redundancy. Flexibility is the ability to deal with new problems while an unforeseen change happens this kind of changes are prevalent in vehicle routing problems when the customer demands are variable due to different factors and make the problem more critical when the products are perishable. Redundancy can be described as the ability to perform an activity with different equipment or manpower, this factor is an important one in vehicle routing and makes the system more reliable.

In this paper, we propose a new multi-objective model which considers a different aspect of a vehicle routing problem with time window which is related and makes the model more practical. To add the redundancy in the model, different kinds of vehicle and manpower are assumed, Flexibility is the other resiliency factor that improves the performance of the model. Considering Reliability, Flexibility and Redundancy makes the system more trustable against unforeseen accidents, reliability can guide the system to avoid high-risk decisions and other factors can improve the performance to return to the normal situation. One of the most important factor parameters in municipal transportations traffic which is able to make an impressive effect on the delivery time. In many real cases traffic has a volatile and non-constant behavior; thus, a stochastic probabilistic time-dependent scheduling is included in the model. To best of our knowledge, this is the first paper that formulated the resiliency and considered it with reliability and green objectives in stochastic space.

Time window problem enforces that each customer should be served within its allowed period. Vehicle capacity problem considers a specific load limitation for each machine to deliver the customer's order (Bahri et al., 2016). The time window assignment vehicle routing problem presented by Spliet and Gabor (2014). Groër et al. (2009) introduced a type of consistent vehicle routing problem that assigned a fixed time window to every customer. Spliet and Desaulniers (2015) studied a discrete time window problem with a set of time windows for every customer. In many cases travel times are assumed to be constant, however; in real situations, travel times are affected by different factors which are volatile variables, thus it is justifiable to formulate a stochastic time-dependent model to assign time windows to customers regarding this feature. Generally, there is not a vide spread literature about TDVRP, this problem was introduced by Malandraki (1989). A time-dependent travel time studied in travel salesman by Cordeau et al. (2012), also Ichoua et al. (2003); Dabia et al. (2013); Franceschetti et al. (2013); Gendreau et al., 2015) have performed different time-dependent researches. A Tabu search algorithm was introduced to deal with TDVRP by Van Woensel et al. (2008)

TWVRP problems are widespread in inventory routing problems and have received lots of attention in recent years. Azadeh et al. (2016a) introduced an inventory routing problem for perishable foods and solved it with the Genetic algorithm. The work accomplished by Coelho \& Laporte (2013) is another inventory routing problem solved by branch and price method. There are different metaheuristic approaches to deal with TDVRP, VRP is among the NP-hard problems (Lenstra \& Kan, 1981). Goksal et al. (2013) proposed a particle swarm optimization (PSO) algorithm, Kuo and Wang (2012) presented a variable neighborhood search (VNS) and Norouzi et al. (2015) showed a simulated annealing (SA) has a better performance rather than (PSO) to deal with TDVRP. 
A good scheduling policy will be useful to handle the traffic issue in municipal transportation and decrease the tardiness of delivery. Rancourt et al. (2013) studied a mix routing and scheduling problem and solved it with a multi-start metaheuristic algorithm that combines adaptive large neighborhood search and mixed integer linear programming. One of the most prominent features of vehicles scheduling is uncertainty to determine the traffic time. A classification of stochastic parameters is proposed by Sahinidis (2004). Stochastic vehicle routing problem (SVRP) considers a probability distribution for random parameters. Gannouni et al. (2017) studied a multi-objective vehicle routing problem and solved it with a probabilistic metaheuristic approach. Sarasola et al. (2016) used a (VNS) method to deal with a stochastic and dynamic VRP problem. In stochastic VRP problems usually to different probabilistic objectives are considered in a model. In this paper time window of costumes is one of the probabilistic parameters which varies due to the seasonal variations in demand. Traffic time is another probabilistic parameter that depends on unforeseen parameters which are predicted by retrospective experiences of the proposed case study. Traffic time can be affected by different accidents such as raining, car accident and other reasons (Duan et al., 2015). There are few studies considering both stochastic and time dependency (Lecluyse et al., 2009; Taş et al., 2014; Sun et al., 2015; Zhang et al., 2016). The green objective is directly associated with the elapsed time in traffic, thus considering this objective together makes sense. Çimen and Soysal (2017) considered a stochastic speed VRP problem with Green objectives. Lin et al. (2014) presented a survey of green VRP trend. Koç and Karaoglan (2016) presented a novel GVRP and solved the problem by a heuristic approach.

Resiliency is a feature that makes a system more flexible and less vulnerable to accidents (Heidari et al., 2017). By decreasing the stress in job atmosphere human resource can get used to the system with better performance (Woods et al., 2012). In recent years reactive safety management is replaced by proactive ones (Azadeh et al., 2017). Resiliency and reliability are taken into account to decrease the probabilistic problems and make the VRP system more agile and flexible, however; we couldn't find many related studies associated with this topic in VRP studies. Ando and Taniguchi (2006) considered reliability in time window while in this paper reliability of trucks has come under scrutiny. Table 1 depicts a summary of recent VRP researches.

\section{Problem description}

This section proposes a modeling basis for the stochastic time-dependent green capacitated vehicle routing and scheduling problem with resiliency and reliability. In the literature, several VRP types are developed. Most of the previous studies have considered a classical objective function, which aims to minimize the total transportation costs between depots and customers. In this study, besides the classical cost function, we include other new objective functions in order to get closer to the real situation. The main assumptions in the presented model are described as follows:

- There is only one depot

- Vehicles speed, capacity, gas emission and price are different

- Traveling with double experts will increase the payments while will decrease the possibility accidents (Experts have the necessary skills in various fields base on resiliency instructions)

- Each customer will be satisfied by one visit

- Traffic time is a random variable with uniform distribution

- Customer demands differ in different seasons and has exponential distribution thus time windows are possibility variables

- Violation of determined time window will increase the spoilage of productions

- There are two types of production with predetermined spoilage possibility which can be decreased by using better transportation equipment's 


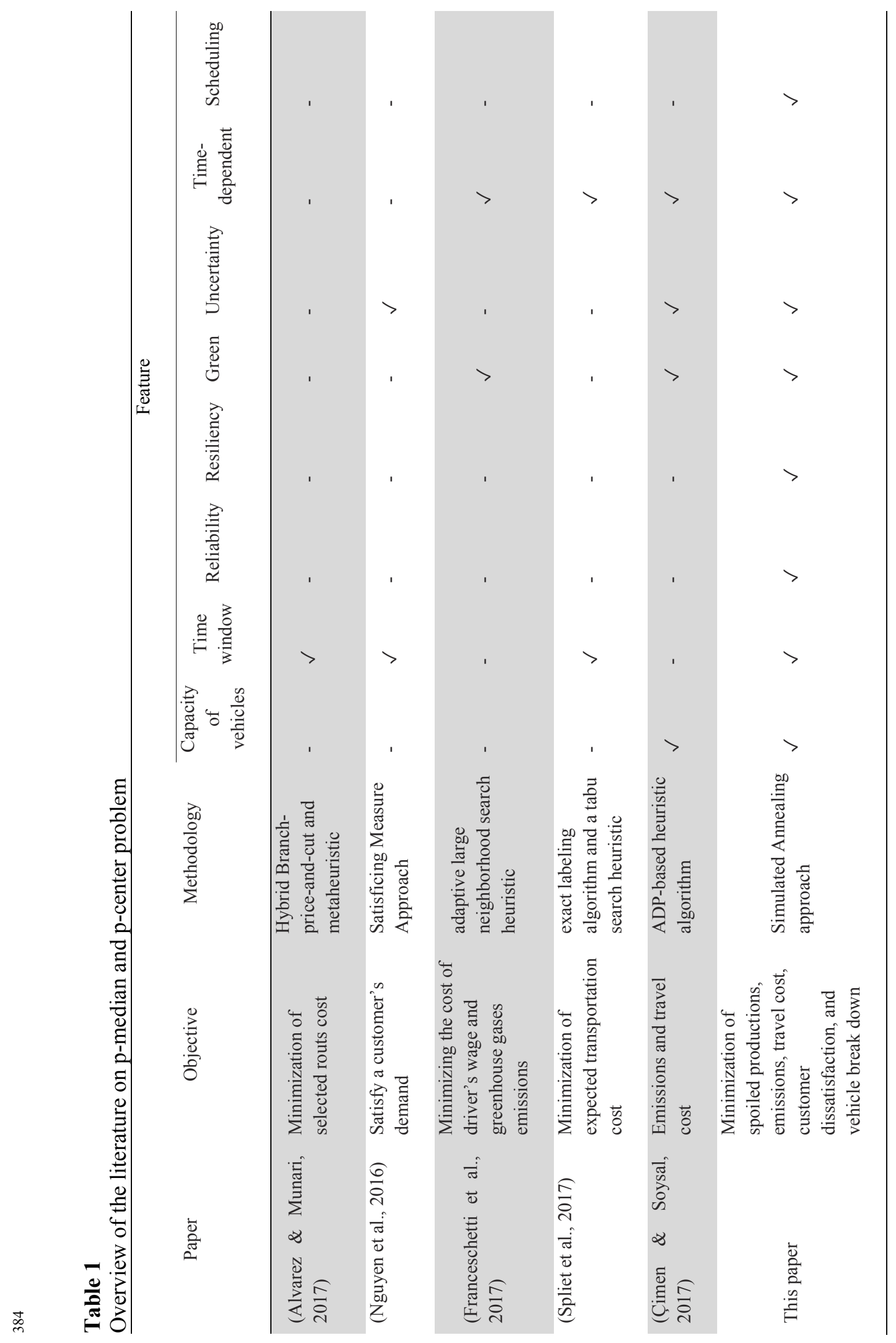


The schematic view of our presented VRP model is demonstrated in Fig. 1.

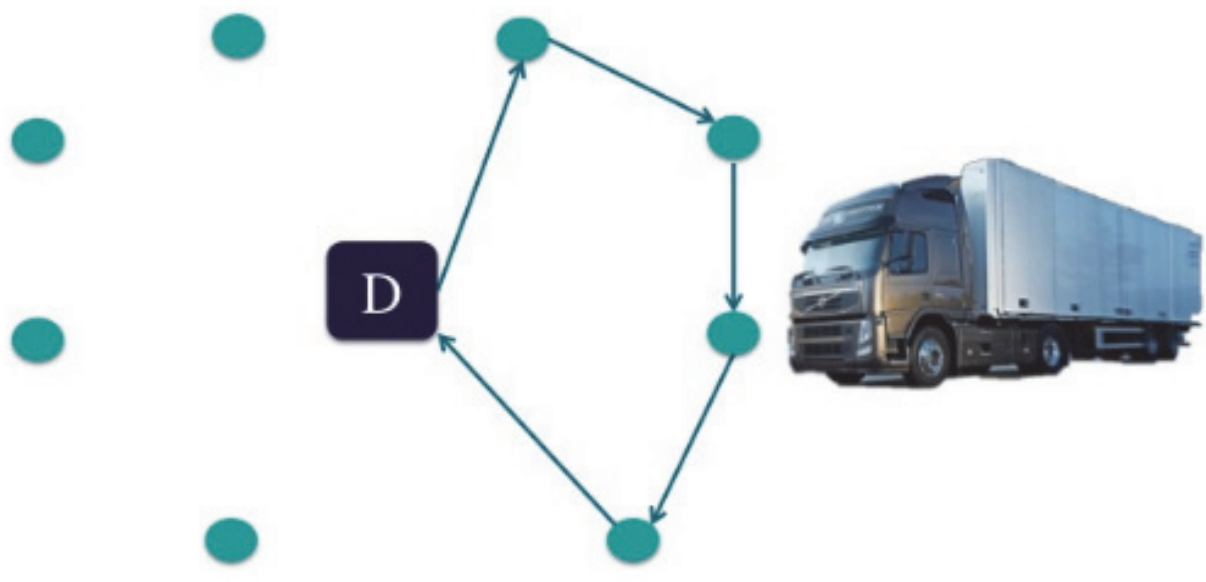

Fig. 1. Proposed VRP Graph

$I \quad(1 \ldots \mathrm{n}) \mathrm{P}$ be a set of clients and depot

$P \quad(1,2)$ be a set of products

$\mathrm{c}_{\mathrm{ijk}} \quad$ be the transportation cost from client $\mathrm{i}$ to $\mathrm{j}$ by vehicle $\mathrm{k}$

$\tilde{c}_{i j k} \quad$ be the transportation cost from client $\mathrm{i}$ to $\mathrm{j}$ by vehicle $\mathrm{k}$ by regarding the presence of added expert

$\mathrm{x}_{\mathrm{ijkt}} \quad 1$ if client $\mathrm{i}$ is assigned to $\mathrm{j}$ by vehicle $\mathrm{k}$ in $\mathrm{t}$ time; and 0 otherwise

$\tilde{x}_{i j k t} \quad 1$ if client $\mathrm{i}$ is assigned to $\mathrm{j}$ by vehicle $\mathrm{k}$ in $\mathrm{t}$ time by regarding the presence of added expert; and 0 other wise

$S P_{p} \quad$ is the spoilage possibility of $\mathrm{p}$-th product

$G H_{k} \quad$ is the Gas emission rate of vehicles

$\operatorname{Cap}_{k} \quad$ is the capacity of each vehicle

$\lambda_{k} \quad$ Break down rate of vehicles per distance unit

$\tilde{\lambda}_{k} \quad$ Break down rate of vehicles per distance by regarding the presence of added expert

$\lambda e r_{t p} \quad$ Customer dissatisfaction rate caused by earliness in time $\mathrm{t}$

$\lambda t r_{t p} \quad$ Customer dissatisfaction rate caused by tardiness in time $\mathrm{t}$

$d_{i j} \quad$ Distance Between customers and depot

dem $_{p t} \quad$ Customer demands of $\mathrm{p}$-th product in $\mathrm{t}$ time

$M \quad$ A big number

$B \quad$ Increasing rate of arrival time

$e_{i} \quad$ earliest time at customer $\mathrm{i}$

$l_{i} \quad$ lates time at customer $\mathrm{i}$

$a_{i} \quad$ arrival time of the delivery vehicle at customer $i$ 


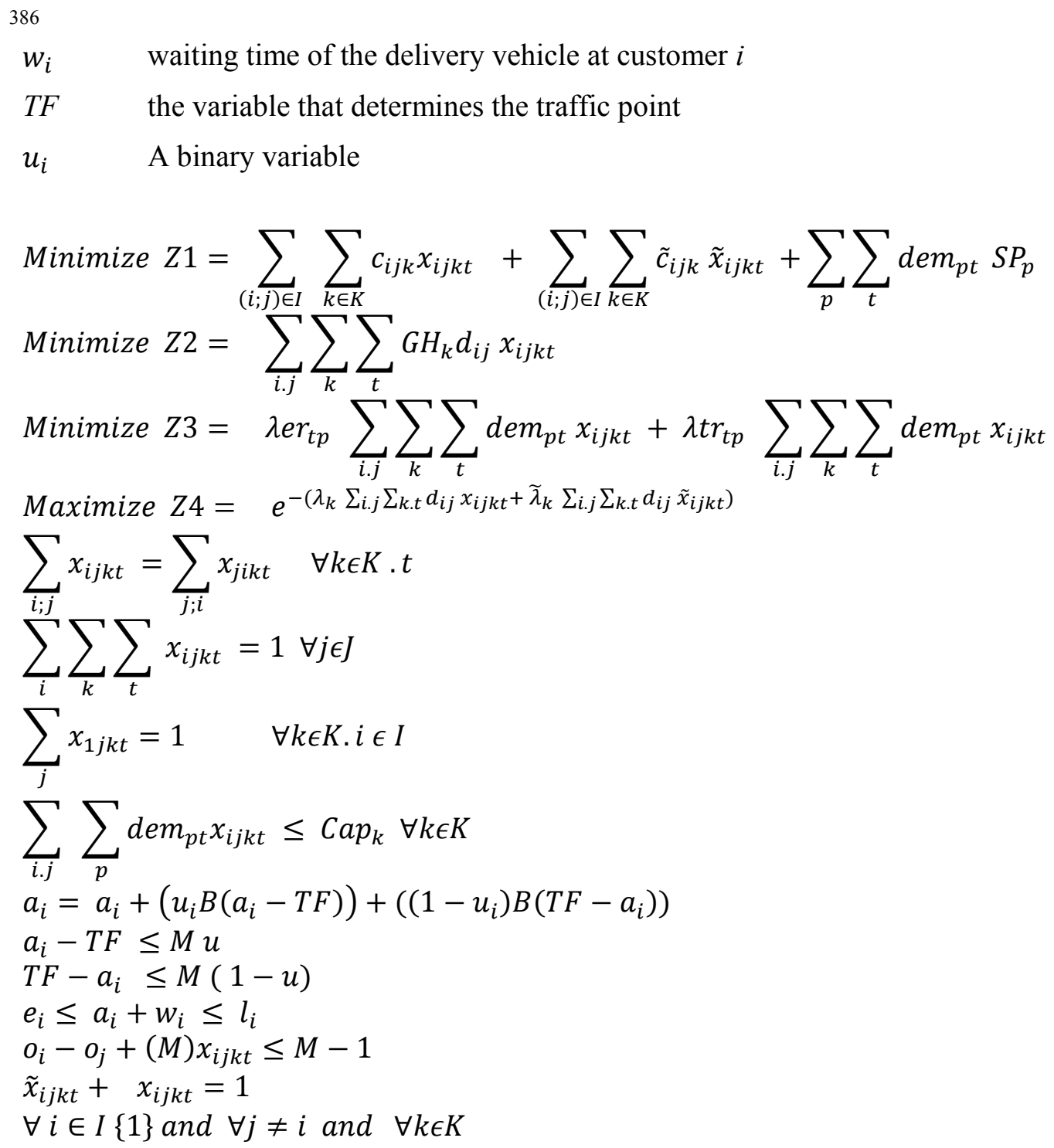

To maximize Z4 it is necessary to minimize the $\left(\lambda_{\mathrm{k}} \sum_{i . j} \sum_{k . t} d_{i j} \mathrm{x}_{\mathrm{ijkt}}+\tilde{\lambda}_{k} \sum_{i . j} \sum_{k . t} d_{i j} \tilde{x}_{i j k t}\right)$. Thus the Z4 objective can change as follows:

$$
\begin{aligned}
\text { Maximize } Z 4 & =e^{-\left(\lambda_{\mathrm{k}} \sum_{i . j} \sum_{k . t} d_{i j} \mathrm{x}_{\mathrm{ijkt}}+\tilde{\lambda}_{k} \sum_{i . j} \sum_{k . t} d_{i j} \tilde{x}_{i j k t}\right)} \sim \text { Minimize } Z 4 \\
& =\lambda_{k} \sum_{i . j} \sum_{k . t} d_{i j} x_{i j k t}+\tilde{\lambda}_{k} \sum_{i . j} \sum_{k . t} d_{i j} \tilde{x}_{i j k t}
\end{aligned}
$$

The objective function (1) is to minimize the transportation cost and spoilage of products. The objective function (2) is included as a green objective to minimize the emitted gas. The objective function (3) minimizes customer's dissatisfaction which varies due to earliness and tardiness and can provide flexibility in decision making. The objective function (4) maximizes the reliability of the system and depends on the type of vehicles and number of experts that are deployed for the proposed destination, this feature can provide redundancy as a critical factor of Resiliency for the model. Constraint (1) is added in order to establish the balance between centers met by vehicles. Constraint (2) force vehicles to meet all the demands. Constraint (3) makes sure that every vehicle starts from the depot. Constraint (4) is about the capacity of each vehicle. Constraints (5)-(7) is about the relation of traffic point and arrival time. Constraint (8) specifies the arrival time of customers. Constraint (9) removes the sub-tours and Constraint (10) enforce to choose between two types of customer meeting. 


\section{Solution method}

The proposed VRP is a possibilistic multi-objective non-linear programming model. In this section, a solution method is proposed to deal with the presented multi-objective under uncertainty. In the first step the non-linear constraints are linearized to make the model as a linear model and in the second step, a procedure is illustrated to deal with an uncertain model. in the third step, the multi-objective model is converted to a single objective. Finally, we have described a method to achieve a feasible solution. The Steps of the proposed hybrid method are demonstrated in Fig. 2.
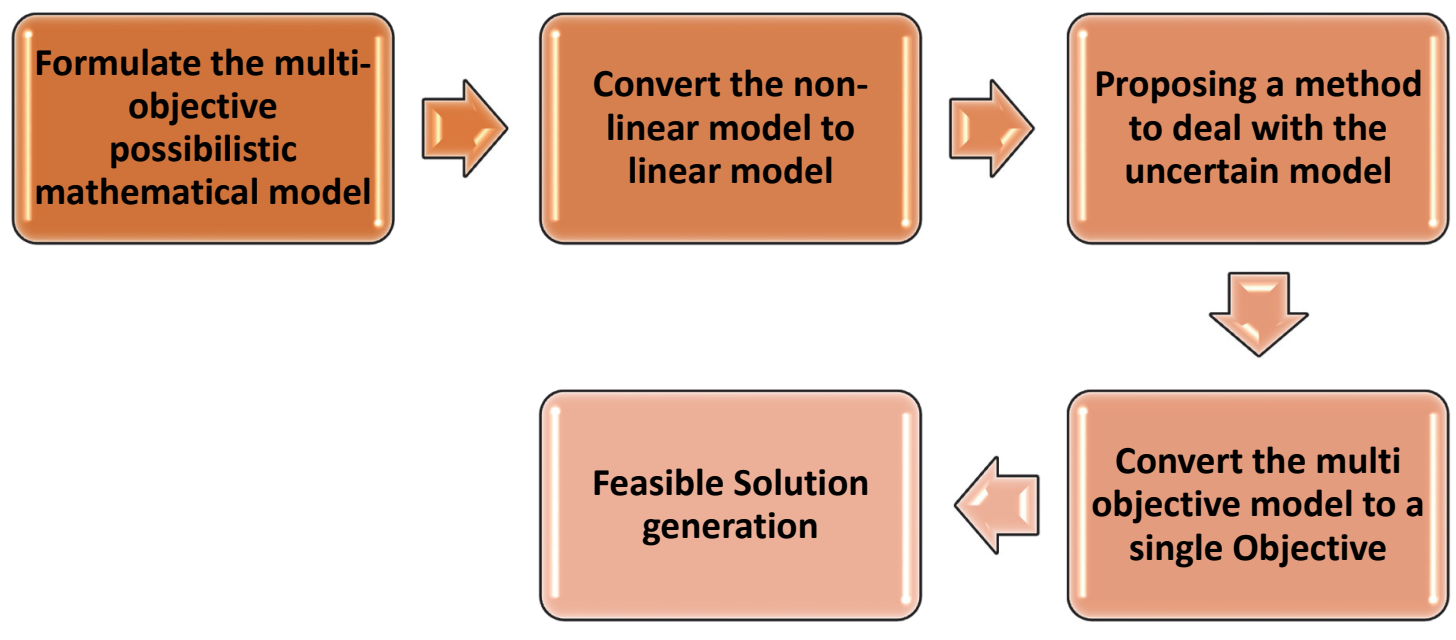

Fig. 2. The steps of the proposed approach

\subsection{Linearizing}

Due to the nonlinear state of constraint (5), this model is a non-linear model. By changing the constraint (5) to a linear one based on Azadeh et al. (2016b), the model will be linearized.

$$
\begin{aligned}
& a_{\mathrm{i}}=a_{\mathrm{i}}+\left(B \pi_{\mathrm{i}}\right)+\left(B \mu_{\mathrm{i}}\right)(11) \\
& \pi_{\mathrm{i}} \leq M u_{\mathrm{i}}(12) \\
& \pi_{\mathrm{i}} \geq\left(a_{\mathrm{i}}-T F\right)-M\left(1-u_{\mathrm{i}}\right) \\
& \mu_{\mathrm{i}} \leq M\left(1-u_{\mathrm{i}}\right)(14) \\
& \mu_{\mathrm{i}} \geq\left(\mathrm{TF}-a_{\mathrm{i}}\right)-M u_{\mathrm{i}}(15) \\
& \mu_{\mathrm{i}} \cdot \pi_{\mathrm{i}} \geq 0
\end{aligned}
$$

\subsection{Uncertainty}

In probabilistic programming, the reliability is the minimum necessity of probability to reach the feasible solution which satisfies constraints. In a standard linear model such as: Min $c^{T} x S \cdot T A x \geq b \cdot x \geq$ 0 where $c$ and $x$ are $n$-vectors, $b$ is an $m$-vector, and $A$ is an $m \times n$ matrix. We have considered a possibilistic term with uncertainity in resources as follow: Min $c^{T} x S \cdot T P(A x \geq b) \geq p \cdot x \geq 0$ where a is deterministic while $b$ is an uncertain variable. In this case (Sahinidis, 2004) illustrated that the probabilistic model is equal to standard linear model.In this paper time windows are variable within the seasons with uniform distribution and traffic points is an exponentional variable, regarding the mentioned study we have considred this variables and solve the model as a linear programing.

\subsection{Multi-Objective model}

Many approaches are introduced to deal with the multi-objective problems such as weighted sum, goal programming, and goal attention. In this paper, a TH method is used, the steps of the TH method can be summarized as follows (Zhalechian et al., 2017): 
Step 1: Set a negative and positive Value for each objective as (NIS) and (PIS)

Step 2: Determine function for each objective function as Eq. (13) for objective functions desired to be minimized and as Eq. (14) for the objective functions desired to be maximized.

$$
\begin{aligned}
& \mu_{\mathrm{k}}(Z)=\left\{\begin{array}{cl}
1 & \text { if } Z_{\mathrm{k}}<Z_{\mathrm{k}}^{\mathrm{PIS}} \\
\frac{Z_{\mathrm{k}}^{\mathrm{NIS}}-Z_{\mathrm{k}}}{Z_{\mathrm{k}}^{\mathrm{NIS}}-Z_{\mathrm{k}}^{\mathrm{PIS}}} & \text { if } Z_{\mathrm{k}}^{\mathrm{PIS}} \leq Z_{\mathrm{k}} \leq Z_{\mathrm{k}}^{\mathrm{NIS}} \\
0 & \text { if } Z_{\mathrm{k}}>Z_{\mathrm{k}}^{\mathrm{NIS}}
\end{array}\right. \\
& \mu_{\mathrm{k}}(Z)=\left\{\begin{array}{cl}
1 & \text { if } Z_{\mathrm{k}}<Z_{\mathrm{k}}^{\mathrm{PIS}} \\
\frac{Z_{\mathrm{k}}-Z_{\mathrm{k}}^{\mathrm{NIS}}}{Z_{\mathrm{k}}^{\mathrm{PIS}}-Z_{\mathrm{k}}^{\mathrm{NIS}}} & \text { if } Z_{\mathrm{k}}^{\mathrm{PIS}} \leq Z_{\mathrm{k}} \leq Z_{\mathrm{k}}^{\mathrm{NIS}} \\
0 & \text { if } Z_{\mathrm{k}}>Z_{\mathrm{k}}^{\mathrm{NIS}}
\end{array}\right.
\end{aligned}
$$

Step 3: In order to convert the multi-objective model into a single-objective one by means of the TH aggregation function. It should be noted that the TH method guarantees to get the efficient solutions (Torabi \& Hassini, 2008). The TH aggregation function is computed by:

$$
\begin{aligned}
& \operatorname{Max} \psi(X)=\vartheta \lambda_{0}+(1-\vartheta) \sum_{k} \varphi_{k} \mu_{k}(Z) \\
& \text { s.t. } \\
& \quad \lambda_{0} \leq \mu_{k}(Z) \quad k=1,2,3 \\
& \quad x \in F(x) \quad \lambda_{0} . \psi \in[0,1]
\end{aligned}
$$

where $F(x)$ indicates the feasible area. Furthermore, $\vartheta$ and $\varphi_{k}\left(\sum_{k} \varphi_{k}=1\right)$ are the coefficient of compensation and the importance of the $k$-th objective function, respectively.

Step 4: Solve the single-objective model. If the DM is satisfied with the gained compromised solution, stop; otherwise, change the values of parameters to obtain another solution.

Feasible Solution Generation:

In order to allocate the customers to vehicles, a randperm generation function is utilized to makes feasible solutions. Let $\{1,5\}$ be the customer numbers and $\{6,7\}$ be the number of the vehicle, then a generated randperm solution would be as presented in Figure 3.

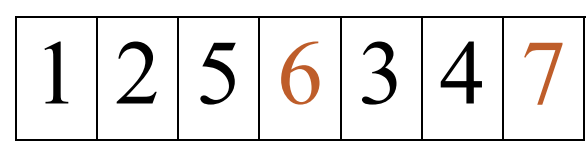

Fig. 3. Solution representation

\subsection{Simulated Annealing}

Simulated Annealing (SA) is a simple and efficient algorithm, based on increasing the iron to its melting point and cool down gradually to the freezing temperatures. The cooler temperature of iron leads to the less molecular movement of the iron particles. In this algorithm, every temperature would be accepted by a specific probability and this possibility decreases over the time. The feature of acceptance probability has made this algorithm less vulnerable to trapping in local optimum solutions. Table 2 shows the summary trend of SA algorithm. 
Table 2

Pseudo code of SA algorithm

Set the parameters

Create initial population

Compute the total cost $\left(\mathrm{TC}_{\mathrm{Vi}}\right)$ for each solution

While stop condition is not satisfied do

a. Select two solutions for displacement

b. Calculate the objective functions of new solutions

c. Generate a random number between $(0,1)$

d. If the Number is less than $\exp (-\Delta \mathrm{z} / \mathrm{T})$ update the T; else go to (a)

If $\mathrm{Z}_{\text {newr }}<\mathrm{Z}_{\text {old }}$ then

End if

Replace the target vector with the new solution vector

End while

\section{Computational Results}

In this section, in order to evaluate the proposed model and the solution method, several sensitivity analyses are done medium-sized test problems. The presented model is run in GAMS 24.7 software and solved using the CPLEX solver. Distribution functions of randomly generated problems are reported in Table 3 based on the Expert judgment of a corruptible food distribution company with 300 crew located in downtown of Tehran. also, other test problems are generated to assess the performance of the proposed algorithm. Notably, all the computations were performed on a laptop with $2.5 \mathrm{GHz}$ CPU and 6 GB RAM. Table 4 shows the parameters of SA algorithm.

Table 3

Source of randomly generated parameters

\begin{tabular}{cc}
\hline Parameters & Values \\
\hline$c_{\mathrm{ijk}}$ & $\sim U(80.100)$ \\
$\tilde{c}_{i j k}$ & $\sim U(85.110)$ \\
$\lambda_{\mathrm{k}}$ & $\sim U(0 \cdot 8.0 \cdot 87)$ \\
$\tilde{\lambda}_{k}$ & $\sim U(0 \cdot 85.0 \cdot 92)$ \\
$\mathrm{M}$ & 100000000 \\
$\lambda e r_{t p}$ & $\sim U(0 \cdot 4.0 \cdot 6)$ \\
$\lambda t r_{\mathrm{tp}}$ & $\sim U(0 \cdot 7.0 \cdot 9)$ \\
$\mathrm{B}$ & $\sim U(0 \cdot 1.0 \cdot 5)$ \\
$d_{i j}$ & $\sim U(1.150)$ \\
$d e m_{p t}$ & $\sim U(10.50)$ \\
$w_{\mathrm{i}}$ & $\sim U(1.5)$ \\
$l_{\mathrm{i}}$ & $\sim U(30.40)$ \\
$e_{\mathrm{i}}$ & $\sim U(1.10)$ \\
$\mathrm{GH}_{\mathrm{k}}$ & $\sim U(0 \cdot 1.0 \cdot 3)$ \\
\hline
\end{tabular}

Table 4

Parameters of SA algorithm

\begin{tabular}{lll}
\hline Algorithm & Parameter & Value \\
\hline SA & T0 & 100 \\
& Alpha & 0.99 \\
& Maximum Iteration & 400 \\
& Inner Maximum Iteration & 20 \\
\hline
\end{tabular}




\subsection{Sensitivity Analysis}

In this section, different test problems are run to evaluate the performance of algorithm compared with GAMS and (PSO). Also, a test with 4 vehicles and 20 customer location is used to evaluate the OFV variations by changing the model's parameters. Gap shows the $\left[100 \times\left(\mathrm{G}_{\mathrm{Opt}}-\mathrm{G}_{\mathrm{Alg}}\right) / \mathrm{G}_{\mathrm{Alg}}\right]$ in large size problems and Gap shows the $\left[100 \times\left(\mathrm{S}_{\mathrm{SA}}-\mathrm{S}_{\mathrm{PSO}}\right) / \mathrm{S}_{\mathrm{PSO}}\right]$.

\section{Table 5}

Computational results of problem

\begin{tabular}{lllllll}
\hline \multirow{2}{*}{ Dataset } & & \multicolumn{2}{l}{ SA } & \multicolumn{2}{l}{ PSO } \\
\cline { 3 - 7 } & $I$ & $K$ & Gap & Time & Gap & Time \\
\hline 1 & 8 & 3 & $0.95 \%$ & 13.8 & $0.95 \%$ & 14.1 \\
2 & 10 & 3 & $1.25 \%$ & 16.6 & $1.25 \%$ & 17.7 \\
3 & 14 & 4 & $1.93 \%$ & 17.2 & $2.05 \%$ & 19 \\
4 & 20 & 4 & $2.38 \%$ & 19.0 & $3 \%$ & 21.4 \\
5 & 25 & 5 & $3.07 \%$ & 23.2 & - & 26.5 \\
6 & 30 & 5 & $3.59 \%$ & 25.7 & - & 28.1 \\
7 & 40 & 6 & $4.30 \%$ & 32.3 & - & 36.5 \\
8 & 50 & 7 & $5.91 \%$ & 38.1 & - & 43.5 \\
9 & 60 & 7 & $6.36 \%$ & 40.5 & - & 46.9 \\
10 & 70 & 8 & $7.25 \%$ & 48.4 & - & 56.9 \\
\hline
\end{tabular}

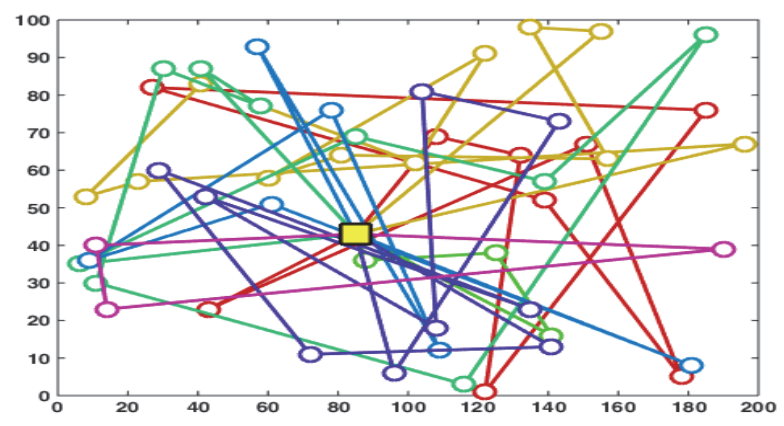

Fig. 4. Execution Result of SA Algorithm

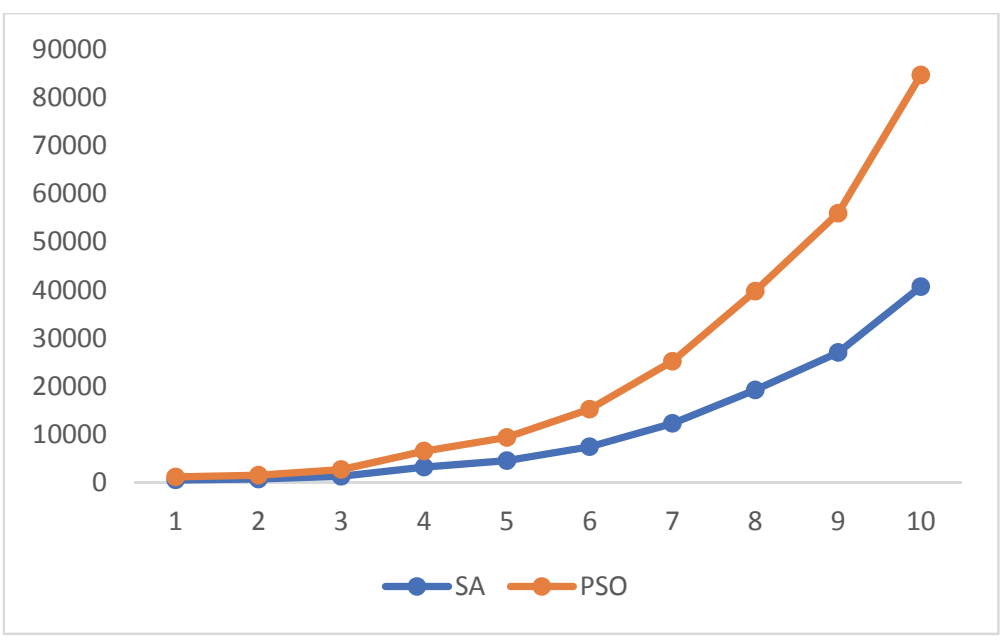

Fig. 5. Runtime Comparison of test problems 


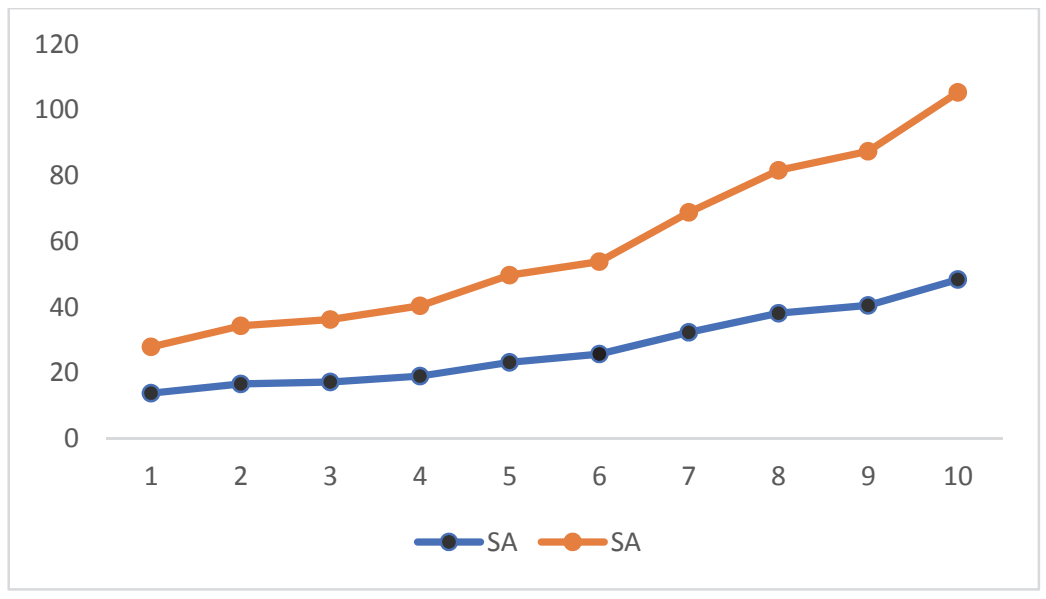

Fig. 6. OFV Comparison of test problems

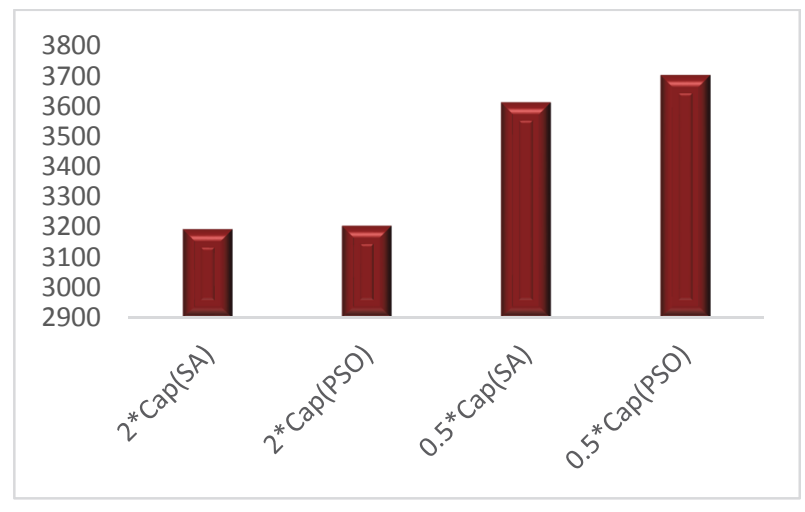

Fig. 7. Impacts of Vehicles Capacity on OFV $(20 * 4)$

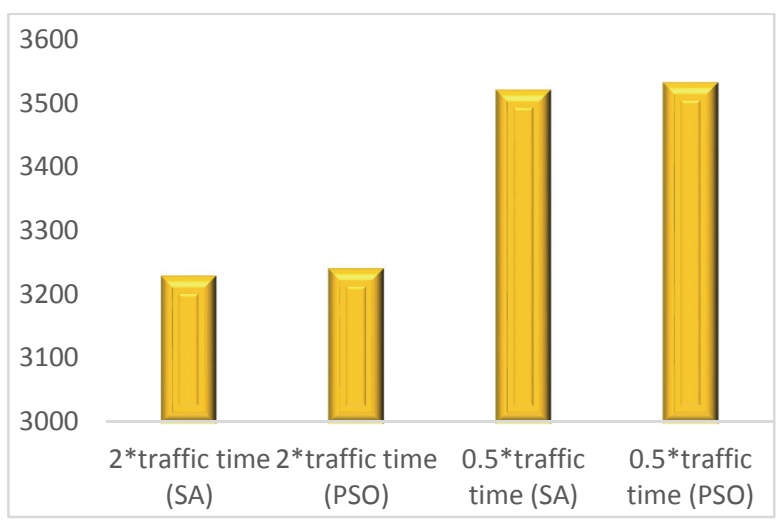

Fig. 8. Impacts of Traffic time on OFV $(20 * 4)$

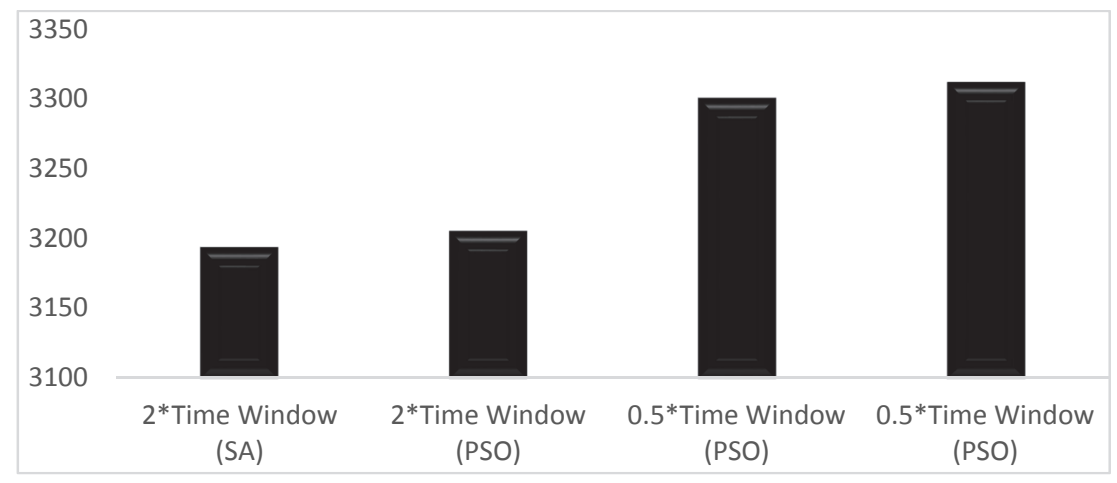

Fig. 9. Impacts of Time window on OFV $(20 * 4)$

\section{Conclusion}

Considering economic, environmental, customer satisfaction and resiliency this paper has presented a multi-objective model to design a stochastic time-dependent green capacitated vehicle routing and scheduling problem with a time window, resiliency, and reliability. In this respect, a scheduling system was used to calculate the arrival time of vehicles at nodes in order to optimize the objectives. To cope 
with uncertain parameters, an efficient stochastic programming approach was applied. Additionally, a hybrid four-phase solution method was proposed to solve the presented model. Furthermore, several numerical experiments and sensitivity analyses were conducted to validate the presented model and the solution method. The results have shown the objective function of the model in different sizes and varying situations. Moreover, the performance of the proposed SA algorithm was evaluated, where the results exhibited the advantage of SA algorithm in comparison to the PSO algorithm.

\section{Acknowledgments}

The authors are grateful for the valuable comments and suggestions from the respected reviewers. They have enhanced the strength and significance of our paper. The authors are grateful for the support provided by the College of Engineering, University of Tehran, Iran.

\section{References}

Alvarez, A., \& Munari, P. (2017). An exact hybrid method for the vehicle routing problem with time windows and multiple deliverymen. Computers \& Operations Research, 83, 1-12 .

Ando, N., \& Taniguchi, E. (2006). Travel time reliability in vehicle routing and scheduling with time windows. Networks and Spatial Economics, 6(3), 293-311.

Azadeh, A., Elahi, S., Farahani, M. H., \& Nasirian, B. (2017). A genetic algorithm-Taguchi based approach to inventory routing problem of a single perishable product with transshipment. Computers \& Industrial Engineering, 104, 124-133.

Azadeh, A, Habibnejad-Ledari, H, Abdolhossein Zadeh, S, \& Hosseinabadi Farahani, M. (2016b). A single-machine scheduling problem with learning effect, deterioration and non-monotonic timedependent processing times. International Journal of Computer Integrated Manufacturing, 1-13 .

Azadeh, A., Yazdanparast, R., Zadeh, S. A., \& Zadeh, A. E. (2017). Performance optimization of integrated resilience engineering and lean production principles. Expert Systems with Applications, 84, 155-170.

Bahri, Oumayma, Amor, Nahla Ben, \& Talbi, El-Ghazali. (2016). Robust routes for the fuzzy multiobjective vehicle routing problem. IFAC-PapersOnLine, 49(12), 769-774 .

Çimen, M., \& Soysal, M. (2017). Time-dependent green vehicle routing problem with stochastic vehicle speeds: An approximate dynamic programming algorithm. Transportation Research Part D: Transport and Environment, 54, 82-98.

Coelho, L. C., \& Laporte, G. (2013). A branch-and-cut algorithm for the multi-product multi-vehicle inventory-routing problem. International Journal of Production Research, 51(23-24), 7156-7169.

Cordeau, J. F., Ghiani, G., \& Guerriero, E. (2012). Analysis and branch-and-cut algorithm for the timedependent travelling salesman problem. Transportation Science, 48(1), 46-58.

Dabia, S., Ropke, S., Van Woensel, T., \& De Kok, T. (2013). Branch and price for the time-dependent vehicle routing problem with time windows. Transportation science, 47(3), 380-396.

Duan, Z., Sun, S., Sun, S., \& Li, W. (2015). Stochastic time-dependent vehicle routing problem: Mathematical models and ant colony algorithm. Advances in Mechanical Engineering, 7(11), 1687814015618631.

Franceschetti, A., Demir, E., Honhon, D., Van Woensel, T., Laporte, G., \& Stobbe, M. (2017). A metaheuristic for the time-dependent pollution-routing problem. European Journal of Operational Research, 259(3), 972-991.

Franceschetti, A., Honhon, D., Van Woensel, T., Bektaş, T., \& Laporte, G. (2013). The time-dependent pollution-routing problem. Transportation Research Part B: Methodological, 56, 265-293.

Gannouni, A., Ellaia, R., \& Talbi, El-G. (2017). Solving stochastic multiobjective vehicle routing problem using probabilistic metaheuristic. Paper presented at the MATEC Web of Conferences.

Gendreau, M., Ghiani, G., \& Guerriero, E. (2015). Time-dependent routing problems: A review. Computers \& operations research, 64, 189-197. 
Goksal, F. P., Karaoglan, I., \& Altiparmak, F. (2013). A hybrid discrete particle swarm optimization for vehicle routing problem with simultaneous pickup and delivery. Computers \& Industrial Engineering, 65(1), 39-53.

Groër, C., Golden, B., \& Wasil, E. (2009). The consistent vehicle routing problem. Manufacturing \& service operations management, 11(4), 630-643.

Heidari, R., Tavakkoli-Moghaddam, R., Yazdanparast, R., \& Aliabadi, L. (2017). A fuzzy data envelopment analysis for the supply chain resilience assessment: An Iranian car manufacturer. Recent Applications of Data Envelopment Analysis, 978(1), 122.

Ichoua, S., Gendreau, M., \& Potvin, J. Y. (2003). Vehicle dispatching with time-dependent travel times. European journal of operational research, 144(2), 379-396.

Koç, Ç., \& Karaoglan, I. (2016). The green vehicle routing problem: A heuristic based exact solution approach. Applied Soft Computing, 39, 154-164.

Kuo, Y., \& Wang, C. C. (2012). A variable neighborhood search for the multi-depot vehicle routing problem with loading cost. Expert Systems with Applications, 39(8), 6949-6954.

Lecluyse, C., Van Woensel, T., \& Peremans, H. (2009). Vehicle routing with stochastic time-dependent travel times. 4OR, 7(4), 363.

Lenstra, J. K., \& Kan, A. H. G. (1981). Complexity of vehicle routing and scheduling problems. Networks, 11(2), 221-227.

Lin, C., Choy, K. L., Ho, G. T., Chung, S. H., \& Lam, H. Y. (2014). Survey of green vehicle routing problem: past and future trends. Expert Systems with Applications, 41(4), 1118-1138.

Malandraki, C. (1989). Time dependent vehicle routing problems: Formulations, solution algorithms and computational experiments.

Nguyen, V. A., Jiang, J., Ng, K. M., \& Teo, K. M. (2016). Satisficing measure approach for vehicle routing problem with time windows under uncertainty. European Journal of Operational Research, 248(2), 404-414.

Norouzi, N., Sadegh-Amalnick, M., \& Tavakkoli-Moghaddam, R. (2015). A time-dependent vehicle routing problem solved by improved simulated annealing. Proceedings of the Romanian Academy Series A-Mathematics Physics Technical Sciences Information Science, 16(3), 458-465.

Rancourt, M. E., Cordeau, J. F., \& Laporte, G. (2013). Long-haul vehicle routing and scheduling with working hour rules. Transportation Science, 47(1), 81-107.

Sahinidis, N. V. (2004). Optimization under uncertainty: state-of-the-art and opportunities. Computers \& Chemical Engineering, 28(6), 971-983 .

Sarasola, B., Doerner, K. F., Schmid, V., \& Alba, E. (2016). Variable neighborhood search for the stochastic and dynamic vehicle routing problem. Annals of Operations Research, 236(2), 425-461.

Spliet, R., Dabia, S., \& van Woensel, T. (2017). The Time Window Assignment Vehicle Routing Problem with Time-Dependent Travel Times. Transportation Science.

Spliet, R., \& Desaulniers, G. (2015). The discrete time window assignment vehicle routing problem. European Journal of Operational Research, 244(2), 379-391.

Spliet, R., \& Gabor, A. F. (2014). The time window assignment vehicle routing problem. Transportation Science, 49(4), 721-731.

Sun, S., Duan, Z., \& Yang, D. (2015). Urban freight management with stochastic time-dependent travel times and application to large-scale transportation networks. Discrete Dynamics in Nature and Society, 2015.

Taş, D., Dellaert, N., van Woensel, T., \& de Kok, T. (2014). The time-dependent vehicle routing problem with soft time windows and stochastic travel times. Transportation Research Part C: Emerging Technologies, 48, 66-83.

Torabi, S. A., \& Hassini, E. (2008). An interactive possibilistic programming approach for multiple objective supply chain master planning. Fuzzy sets and systems, 159(2), 193-214.

Van Woensel, T., Kerbache, L., Peremans, H., \& Vandaele, N. (2008). Vehicle routing with dynamic travel times: A queueing approach. European journal of operational research, 186(3), 990-1007.

Woods, D. D., Leveson, N., \& Hollnagel, E. (2012). Resilience engineering: concepts and precepts: Ashgate Publishing, Ltd. 
Zhalechian, M., Tavakkoli-Moghaddam, R., \& Rahimi, Y. (2017). A self-adaptive evolutionary algorithm for a fuzzy multi-objective hub location problem: An integration of responsiveness and social responsibility. Engineering Applications of Artificial Intelligence, 62, 1-16.

Zhang, J., Lam, W. H., \& Chen, B. Y. (2016). On-time delivery probabilistic models for the vehicle routing problem with stochastic demands and time windows. European Journal of Operational Research, 249(1), 144-154.

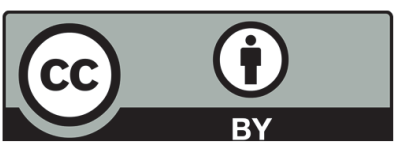

(C) 2018 by the authors; licensee Growing Science, Canada. This is an open access article distributed under the terms and conditions of the Creative Commons Attribution (CC-BY) license (http://creativecommons.org/licenses/by/4.0/). 